\title{
Safety and health leadership: a concise 'story line' to achieve zero harm
}

\author{
by K. de Jager
}

\section{Synopsis}

A holistic safety and health approach is necessary for a company to succeed in achieving zero harm. Potential systemic failures are highlighted that could exist within organizations, which create the 'error traps' where accidents become inevitable. Safety and health leadership strategies and the interdependencies between leadership components and health and safety systems are described to understand 'cause and effect'. Human factors and human error in health and safety, as well as the application of behavioural safety theory and methods, help to look at people in context, both within and outside the workplace.

Keywords

safety, leadership, human factors, human error, human behaviour, safety culture. fit together as a whole in order to achieve zero harm.

As a start, the leadership of a company must instill zero harm as a non-negotiable value for the company. However, it should be understood that zero harm is a reactive mindset in that it implies that an incident is expected to occur, but that the consequences should result in zero harm. The next desirable proactive mindset is that of 'zero incidents', meaning that all risk management controls are appropriate and applied.

Genuine caring for people creates an environment conducive for zero harm. 'Values' on a wall poster that are not being applied, especially by leadership, have significant negative consequences on the safety culture in the longer term. Management must be seen to live the values and lead by example.

The company's safety efforts should be documented and contained in a safety management system (SMS), agreed to by all and applied by all stakeholders in the company. Participative management is important to the success of the 'zero harm' concept.

A credible dynamic risk management process must underpin the SMS. Risks at all levels of the organization must be identified and controls instituted. Controls must be applied by all so as to ensure that activities are within the company's residual risk tolerances.

Safety is a result of proactive risk management and is a concurrent activity to ensure that controls are in place before and during the execution of any task and not something that is done subsequently. For every task to be performed there should be an agreed safety procedure available and all staff should agree to work to it.

\footnotetext{
* Visiting Adjunct Professor, School of Mining Engineering, University of the Witwatersrand, South Africa.

(C) The Southern African Institute of Mining and Metallurgy, 2018. ISSN 2225-6253. Paper received Mar. 2017; revised paper received Jun. 2017.
} 


\section{Safety and health leadership: a concise 'story line' to achieve zero harm}

What gets measured gets done. The safety information system must be appropriate for the company, with leading and lagging indicators which are relevant, accurate, and available timeously. More importantly, appropriate action should be taken should indicators trend negatively.

Human factors, which include the theory of behaviour as well as human errors, should be well understood, including human limitations. This will include the behaviour of leadership as well as the individual who is in the line of fire. Behaviour turns systems and procedures into reality.

Benchmarking of relative industry performance should be undertaken and system audits should be done regularly to ensure compliance of the company's safety policy. The company should strive for continual improvement of its safety performance.

The abovementioned is a high-level safety story line that will be unpacked in more detail in the rest of the paper.

\section{Safety leadership}

The role of leadership is to enable, influence, and to motivate others towards achieving the same purpose goals contributing to the effectiveness and success of the organization or group of which they are members.

Leadership must embrace the notion that all injuries are preventable and that no injury is acceptable. Safety is a priority and schedule, cost, or production will not take precedence over an injury-free workplace.

Studies in safety leadership indicate that the behaviours of supervisors and senior managers have a direct influence on worker behaviours and thus on workplace health and safety. Safe behaviours at work are influenced by specific leadership approaches. Senior managers are the key influencers on the safety culture of an organization and effective safety leadership is vital to the success of a safety programme. Management's safety leadership lays the foundation upon which a solid system is built.

\section{Lead by example}

Leadership determines whether safety will be achieved in an organization through its actions, commitment, measures, example, and rewards.

Effective safety leadership determines the extent to which safety rules and procedures are adhered to in reality. Top management's commitment is expressed through action, not only talk. Example and integrity have been noted as supreme qualities of leadership.

Leaders demonstrate safety when the words and deeds match. If you say 'be safe' and act in ways that do not ensure safety, it sends out conflicting messages. Good example and integrity build good faith, trust, and confidence.

\section{Leadership style}

Management attitude, behaviour, and style tend to be less quantifiable indicators of safety management outcomes than safety management systems. However, these factors have a very powerful effect on workforce safety and help separate the excellent organizations from the merely good ones.

Legislation, in most countries, provides a basis for worker representatives to provide input into decision-making that affects worker safety. People work more safely when they are involved in the decision-making process, have specific and reasonable responsibilities, and have immediate feedback about their work.

While it is recognized that regulation is necessary for good safety, compliance with legislation and a regulatory regime alone do not lead to an outstanding safety record. However, this commitment must extend beyond satisfying regulatory requirements to achieving improvements in safety management

Some of the obstacles to effective safety leadership include:

> Safety is thought of as only a system of discipline

> Safety efforts are delegated to lower levels in the organization and leaders have little hands-on involvement

> Passively handing over safety leadership to external agencies or companies

> Lack of participation, poor communication, lack of accountability, lack of enforcement, lack of clarity, 'Us $v s$. Them' mentality

> Safety as a priority is never demonstrated, just talked about.

\section{Organizational culture}

An organizational culture does not spring up ready-made. Organizations, like organisms, adapt. Safety cultures evolve gradually in response to local conditions, past events, the character of the leadership, and the mood of the workforce (Reason, 1998).

The degree to which control over work organization and task structure is centralized is an important consideration in the culture of safety, with greater decentralization making for better safety outcomes.

\section{Safety culture}

A safety culture is the product of individual and group values, attitudes, competencies, and patterns of behaviour that determine commitment to safety (Booth, 1996). It is something an organization is, the beliefs, attitudes, and values of its members regarding the pursuit of safety. It is also something that an organization has, the structure, practices, controls, and policies designed to enhance safety. It is the way a company thinks and acts towards safety and it is believed to be a key predictor of safety performance.

A safety culture consists of shared values - what is important, and beliefs - how things work to produce behavioural norms ('the way we do things around here') (Strong, 2012). A positive safety culture is where there are trustful communications, shared perceptions of importance of safety, and confidence in the efficacy of preventive measures (Booth, 1996). Changing workplace culture may be difficult, but its success is very much dependent on good leadership.

\section{Ideal safety culture}

An ideal safety culture is the 'engine ' that drives the system towards the goal of sustaining the maximum resistance towards its operational hazards, regardless of the leadership's personality or current commercial concerns.

The power of this engine relies heavily on a continuing respect for the many entities that can penetrate, disable, or bypass the system's safeguards (Reason, 1998). Safety is invisible in the sense that safe outcomes do not deviate from 


\section{Safety and health leadership: a concise 'story line' to achieve zero harm}

the expected, and so there is nothing to capture the attention. If people see nothing, they presume that nothing is happening, and that nothing will continue to happen if they continue to act as before. But this is misleading because it takes a number of dynamic inputs to create stable outcomes (Reason, 1998).

\section{Safety climate}

A safety climate is a subset of safety culture concerned with the values and attitudes of staff at all levels (Booth, 1996). Although a company may have an ideal safety culture, the safety climate or 'mood' may be depressed if there is a serious injury, for example.

\section{Safety management systems}

In the health and safety literature, systems are referred to frequently as being composed of humans, machines, and the environment, which interact in order to achieve a defined goal and have the following characteristics:

> Systems are purposive

- They can be hierarchical, that is, considered to be parts of larger systems or a 'nesting' of systems within a set system boundary

> They operate in an environment

> Systems components serve functions - information receiving, information storage, information processing and decision, and action

> The components of a system interact

> Systems, sub-systems, and components have inputs and outputs (Sanders and McCormick, 1993).

A SMS is a set of plans, actions, and procedures to systematically manage safety in the workplace that is actively endorsed by a committed employer to achieve the following:

> Provision of a safe and healthy workplace and the prevention/reduction of illness and injury for both employees and contractors

> Identification of workplace hazards, assessment and control of all risks

> Active involvement in health and safety matters by managers, supervisors, and employees and their representatives

> Provision of information and training for employees at all levels so they can work safely.

It is important to put effort into continuous improvement, which is building in ways of periodically checking how you are doing and how to improve your system, using feedback mechanisms, audits, and inspections.

Developing a structured training strategy, with the ability to meet different language and education needs, is a significant factor to attaining success. An effective system is more than a paper chase. It is about making sure that relevant changes occur, to improve safety. However, documenting what you do is good business practice.

\section{Why safety management systems succeed}

The SMS is directly aimed at managing critical risks. Senior managers or owner/operators practice what they preach and make decisions to eliminate or reduce hazards. Supervisors and employees understand their role in the system and are closely involved in its development and operation.
The system is commensurate with the risks to be managed and is absolutely tailored to the organization's requirements. The SMS is able to withstand, and welcomes, robust questioning and review from everyone involved.

\section{Why safety management systems fail}

> Management support is irregular and inconsistent

- Everyone treats the system as 'paper warfare' and follows procedures to keep the managers happy, regardless of their effectiveness

> The SMS is established in response to external demands and never really 'owned' or understood by those subject to it

> The system is imposed without effective participation by those who have to make it work

> The effort to build an organization-specific system is not made and 'off the shelf' solutions are applied that have little relevance to the culture of the workplace

- The SMS lacks the full support of, and commitment from, management

> Due diligence is not shown, for example, by having safe work practices and procedures that sit, fully documented, in a folder on the shelf - but are never used

> The performance of safety and health as well as injury management systems should be continually reviewed and opportunities identified for improvement.

Those who manage and operate the system must have current knowledge about the human, technical, organizational and environmental factors that determine the safety of the system as a whole. For an accident to occur in such a system, it requires the unlikely combination of several different factors to penetrate the many protective layers and to allow hazards to come into damaging contact with plant, personnel, and the environment (Reason, 1998).

Some of the barriers to the effective implementation of safety and health management systems are:

> Failure to meet necessary conditions (for example, by not being customized to the needs of the organization)

> Inappropriate use of audit tools (for example, where they become an end in themselves)

> Application in hostile environments (for example, in areas with precarious employment).

Make sure leaders in the organization are familiar with the SMS. Recognize that cultural and attitudinal change may be necessary to improve performance, and that this takes time.

\section{Risk management}

The principles for preventative and protection measures include avoiding a risk altogether, or using a different approach, substance, or method of work. Risks should be combated at source rather than by adopting secondary measures. Work should be adapted to the individual rather than expecting the individual to adapt to the work.

When treating risks, advantage should be taken of technological and technical progress. Risk prevention measures must be part of a coherent policy and approach to safety management. 


\section{Safety and health leadership: a concise 'story line' to achieve zero harm}

The causation model (Figure 1) depicts a systematic analysis of potential multiple causes of incidents. The model is roughly based on the previous work done by Bird and Heinrich (1985).

In the causation model, risk management $v s$ crisis management, proactive $v s$ reactive, from paper to practice, as well as leadership, errors, and behaviour is depicted. Another example is the Swiss cheese model (Figure 2), where risk management barriers are discussed.

Risk barriers should consider a four-stage approach:

> Prevention barriers intended to prevent the energy from getting out of control (with adequate reinforcement or redundancy where barrier failure is probable)

> Monitoring barriers intended to monitor the status of the energy to identify if it is different from expected and if the barriers are as effective as required

> First-response barriers intended to stop an unwanted event in the early stages before any significant consequence can occur

> Amelioration barriers intended to minimize the consequences of a major unwanted event (NSW Mine Safety Operations Branch, 2011).

\section{Safety information system}

It is difficult for leaders to make an informed contribution to safety and injury management without an understanding of the relevant regulatory frameworks, individual, responsibilities, or emerging health, safety, and injury management risks and issues.

In the absence of frequent unwanted events, the best way to induce and then sustain a state of intelligent and respectful wariness is to gather the right kinds of data. This means creating a safety information system that collects, analyses, and disseminates information from incidents and nearmisses, as well as from regular proactive checks on the system's vital signs. (Reason, 1998)

\section{Criteria for selecting indicators}

> Indicators should be worth measuring

- Indicators can be measured for diverse populations

> Indicators can be understood by people who need to act

> Information will galvanize action

- Actions that can lead to improvement are known and feasible
Measurement over time will reflect the results of action (Chrvala and Bulger, 2010).

\section{Performance and outcome indicators}

Using a mix of positive performance or lead indicators and outcome or lag indicators will help organizations achieve targets and improve performance. A supplementary view considers process indicators, which refer to asset or technical integrity, and personal indicators, which refer to personal injury (Hopkins, 2007).

Positive or lead performance indicators allow an organization to measure activities undertaken that are designed to impact positively on outcome or lag performance. Leading indicators are predictive, require active monitoring, and provide feedback on performance before an accident or incident (HSE, 2006)

Outcome indicators are important measures, but generally reflect the results of past actions. There is often a time lag between an action taken by an organization to improve performance and any measurable change in performance.

Outcome indicators may hide potential risks. For example, a low incidence of injury does not necessarily mean that adequate safety systems and controls are in place. Therefore relying on lag indicators to gauge a company's safety performance is problematic, as this approach is after the fact and measures failures. Reactive monitoring involves identifying and reporting on incidents to check whether the controls in place were adequate, to identify weaknesses or gaps in control systems, and to learn from mistakes (HSE, 2006).

\section{Human factors}

The aim of applying human factors is to optimize the performance of individuals and teams in the workplace,

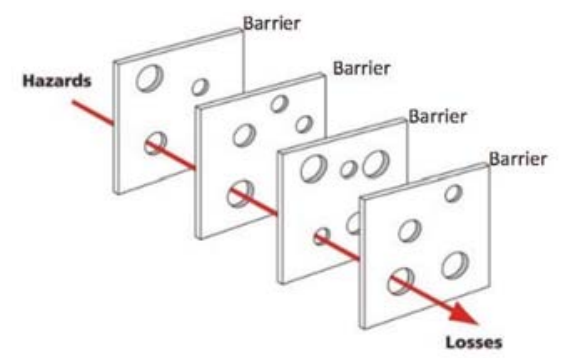

Figure 2-Reason's Swiss cheese model

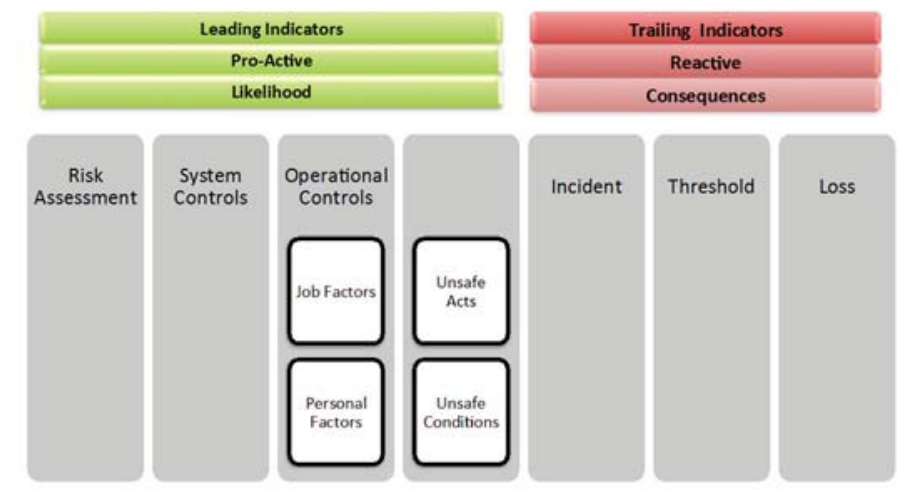




\section{Safety and health leadership: a concise 'story line' to achieve zero harm}

reduce error, and improve safety and efficiency through an understanding of human capabilities and human limitations.

A human factor is any biomedical or psycho-social consideration relating to human characteristics in areas including, but not limited to, human engineering, humanmachine interface, personnel selection, training, job performance, and human performance.

Human factors is an applied science that draws on methods and principles from psychology and other behavioural and social sciences, engineering, ergonomics, and physiology.

The human factors domain includes communication, knowledge, skills, abilities, procedure use, workload on the human, fatigue, situational awareness, attention, and safety

The role of human factors in management system design, evaluation, and implementation is critical. With increasing automation and advanced technology it is important to identify and manage human performance-related issues as early as possible. This will result in optimized relationships between people, tasks, technologies, and the working environment aimed at ensuring safe and efficient human performance (Eurocontrol, 2008). Human factors integration enables designers to understand what end-users can and cannot do as compared to the tasks they will be required to undertake once the 'system' is operational.

There is a wide body of knowledge on human capabilities and both physical (e.g., body size, tolerances, strength) and mental (e.g., perception and information processing capabilities) limitations (Cullen, 2007).

\section{Behaviour}

Almost 90 years of research and observation, ranging from Heinrich's early hypothesis in the 1930s to DuPont's timetested successes of today, confirm that unsafe behaviours are involved in almost all accident occurrences. It could also be argued that unsafe behaviours in the boardroom, regarding making inappropriate decisions on safety-related issues, could also be the cause of an incident.

The core questions remain: Why do employees do what they do, act unsafely, and have accidents? What 'causes' atrisk behaviour? (Hansen, 2007),

Good safety systems on their own do not ensure successful health and safety management; the level of success is determined by how organizations 'live' their systems (Reason, 1998).
There is evidence that a proportion of accidents are caused by unsafe behaviour. Promoting safe behaviour at work is a critical part of the management of health and safety, because behaviour turns systems and procedures into reality. At-risk behaviour is the critical element, which must be addressed to achieve safety excellence.

Changing unsafe behaviour into safe behaviour is appropriate; however, this should not deflect attention from analysing why people behave unsafely. Behaviour modification techniques can be effective in promoting critical health and safety behaviours, provided they are implemented effectively.

Focusing on only treating individual behaviour without considering necessary changes to how people are organized, managed motivated, rewarded, their physical work environment, and tools and equipment can result in treating the symptom only, without addressing the root causes of unsafe behaviour (HSE, 2002).

\section{Human error}

It is important to focus on human error (Figure 3) so as to reduce vulnerability to human error, i.e. prevent accidents, to understand the trade-offs and pressures which people encounter at work, and to clarify the value and limitations of human error theory.

The expression 'human error' is widely used and generally misused. It has been traditionally associated with the attribution of responsibility and blame. In this context, 'human error' is typically a judgement of human performance made after an event has occurred (Massaiu, 2005).

Human error and 'pilot error' are terms used synonymously, and in the aviation industry it is an overly simplistic, if not naive, approach to accident causation. The same applies to the assigning of accidents to 'operator error' in other industries. Accidents cannot be attributed to a single cause, or in most instances, even a single individual. Even the identification of a 'primary' cause is fraught with problems. Accidents are the end result of a number of causes, only the last of which are the actions ('unsafe acts') of the aircrew or the operators (Wiegmann, 2000).

A poor safety culture is likely to increase the number of defensive weaknesses due to active failures. Front-line errors are more likely in organizations that are insufficiently concerned about the working conditions known to promote the slips, lapses, and mistakes of both teams and individuals.

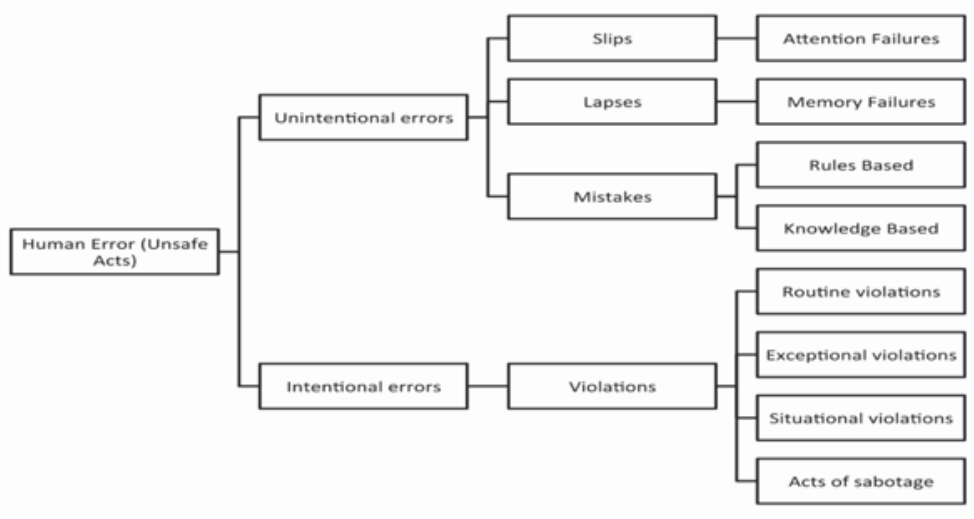




\section{Safety and health leadership: a concise 'story line' to achieve zero harm}

These include such factors as inadequate training, poor communication, poor procedures, and problems with the design of the human-machine interface. But, perhaps even more potently, a poor safety culture will encourage an atmosphere of noncompliance with safe operating practices.

Violations are likely to be most common in organizations where the unspoken attitudes and beliefs mean that production and commercial goals are seen to outweigh those relating to safety.

The effect of a poor safety culture is evident in the unwillingness to deal proactively with known deficiencies in the defenses. In short, defensive gaps will be worked around and allowed to persist. The history of organizational accidents is rich with examples of management neglecting or postponing the elimination of previously identified defensive weaknesses (Reason, 1998).

\section{Safety maturity}

The safety maturity journey of a company can be characterized in various ways using safety maturity models. Figure 4 is an example described by Ryder Marsh (Strong, 2012)

The DuPont Bradley curve (Figure 5) is another way of depicting the shifts in mindset and actions that need to occur over time to develop a mature safety culture. The safety maturity journey is as follows: a reactive culture, based on natural instincts; a dependent culture, based on supervision; an independent culture, based on self; and a final interdependent culture based on a team approach.

An accident is an undesirable or unfortunate happening that occurs unintentionally and usually results in harm, injury, damage, or loss; casualty; mishap: or vehicle accident. It includes the entire interacting circumstantial framework (chance, pre-existing, or uncontrolled dynamically developing conditions such as commonplace actions, random time and place, participants etc.) leading up to, including, and resulting from, the accident's immediate occurrence.

Other models include the Hudson Ladder model (SIEP, 2003) and the minerals industry risk management maturity chart (NSW Mine Safety Operations Branch, 2011).

\section{Hidden and indirect safety costs}

While workers' compensation costs (typically paid as premiums) are a significant component of the cost of workplace injury and disease, there are other hidden and indirect costs. For example, for employers they include:

\section{- Lost productivity}

> Loss of skills, experience, and knowledge
> Cost of recruitment, replacement and training

> Increased workload pressure and uncertainty for coworkers

> Higher risk of injuries to other staff and lowered morale

> Cost of replacement equipment

> Damage to the organization's reputation and image as an attractive workplace

> Cost of investigations and reports.

\section{Conclusions}

Although a significant amount of information is available globally with respect to safety, it is mostly focused on specific aspects of a 'safety management system'. The intent of this paper is to clarify the context within which interested parties share concepts and contribute to the enhancement of the overall safety process.

\section{Acknowledgments}

This paper was adapted from a previous paper published in 2015 at the Annual Meeting and Conference of the Society of Mining Professors, Freiberg, Germany.

Thank you to the University of the Witwatersrand and the Society of Mining Professors for allowing me to republish this paper.

\section{References}

BiRD, F.E.J. and Germain, G.L. 1985. Practical Loss Control Leadership. International Loss Control Institute Inc., Loganville, GA.

Воотн, R.T. 1996. The promotion and measurement of a positive safety culture. Human Factors in Nuclear Safety. Stanton, N.A. (ed.). Taylor \& Francis.

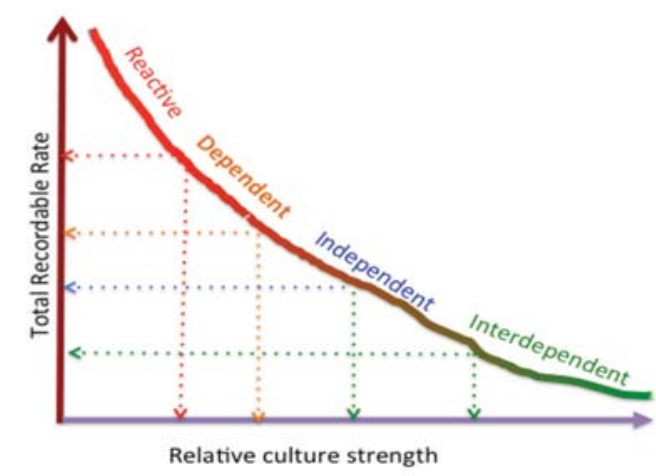

Figure 5-DuPont Bradley curve

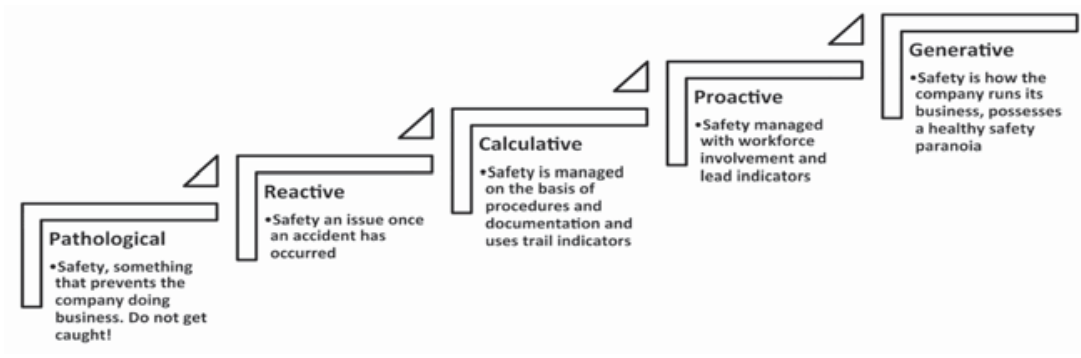

Figure 4-Safety culture maturity model 


\section{Safety and health leadership: a concise 'story line' to achieve zero harm}

CuLLEN, L. 2007. Human factors integration - Bridging the gap between system designers and end-users: A case study. Human Engineering Limited, Westbury on Trym, Bristol, UK.

Chrvala, C.A. and Bulger, R.J. (eds.). 2010. Leading Health Indicators for Healthy People 2010: Final Report. National Academy Press, Washington, D.C.

EuRoconTrol. 2008. Safety Culture in Air Traffic Management. European Organisation for the Safety of Air Navigation.

Hopkins, A. 2007. Thinking about process safety indicators. Proceedings of Chemistry in the Oil Industry XV, Manchester, 6-8 November. Royal Society of Chemistry.

HSE (UK Health and Safety Executive). 2006. Developing process safety indicators: a step-by-step guide for chemical and major hazard industries.

HSE. (UK Health and Safety Executive). 2002. CRR 430/2002. Strategies to promote safe behaviour as part of a health and safety management system.

HANSEN, L. 2007. A universal model for safety excellence. Occupational Hazards. http://www.ehstoday.com/safety/ehs_imp_70197

HeInRich, H.W. 1931. Industrial Accident Prevention, A Scientific Approach. McGraw-Hill.
MASSAIU, S. 2005. Human error and models of behaviour. IFE/HR/E-2005/031. IFE Institute for Energy Technology, Kjeller, Norway.

NSW Mine Safety Operations BRAnCh. 2011. MDG 1010: Minerals industry safety and health risk management guideline. NSW Department of Industry and Investment, Sydney, Australia.

NorTHERn Territory Government. 2012. Safe Work Australia. 2012. Safety Management Systems. Guide for Major Hazard Facilities. Darwin, NT

REASON, J.1998a. Achieving a safe culture: theory and practice. Department of Psychology, University of Manchester, UK.

REASON, J. 1998b. Managing the risks of organisational accidents. Ashgate, Aldershot.

SANDERS, M.S. and McCoRmick, E.J. 1993. Human factors in engineering and design. McGraw-Hill.

SIEP BV. 2003. Winning Hearts and Minds: A Road Map. Shell International, Rijswijk, The Netherlands.

Strong, M. 2012. Proceedings of the IOSH IPD/CPD Workshop, Netherwood Hotel, Grange-over-Sands, Cumbria. Institution of Occupational Safety and Health, Leicester, UK.

Wiegmann, D.A. 2000. The human factors analysis and classification SystemHFACS. FAA Civil Aeromedical Institute, Oklahoma City, OK. 